\title{
L'accès à la justice et ses obstacles
}

\author{
Par René Degni-Segui
}

"Il faut que l'accès du prétoire soit relativement aisé pour les justiciables ... C'est là une condition d'une bonne justice" ${ }^{1}$. Cette réflexion de René Cassin nous fournit d'emblée la problématique de notre étude, à savoir la dialectique entre le justiciable et la justice. Celle-ci est faite pour celui-là, nous rappelle le Professeur Rivero, en ce que "la justice est un service public dont la raison d'être est l'usager". Telle est l'"évidence propre à se dérober aux yeux du spécialiste" et que l'éminent juriste se fait fort de nous recommander "de garder sans cesse à l'esprit" ${ }^{2}$.

Cette évidence se traduit concrètement d'abord et avant tout par l'accès du justiciable au prétoire pour faire entendre sa cause. Car, faute de pouvoir y accéder, l'usager du service public ne peut faire valoir ses droits et la justice elle-même serait un vain mot, une justice sans justice, en définitive un déni de justice. Il suit de là que l'accès à la justice est une condition de réalisation des "droits à la justice", tels le droit à un procès ou les droits garantis à l'accusé (présomption d'innocence, légalité des délits et des peines, autres droits de la défense...). Il constitue lui-même un droit fondamental, celui de disposer d'un recours devant l'autorité compétente et ici l'autorité judiciaire. L'on comprend dès lors l'importance que revêt l'étude des obstacles susceptibles de paralyser, de bloquer ou même de restreindre l'accès à la justice et rendre ainsi ineffectif le droit qui en dérive.

La réalisation du droit à la justice en Afrique noire francophone est influencée par deux paramètres agissant en sens contraire, l'un favorable, résultant de la garantie dudit droit dans son existence ou sa jouissance, et l'autre défavorable, se traduisant par son exercice conflictuel avec les droits africains.

Le droit de recourir à la justice, hérité du droit colonial, a été naturalisé et garanti dans sa jouissance. Il bénéficie en effet d'une triple protection universelle, régionale et nationale. Au plan universel, la plupart des Etats africains, parties à la Charte des Nations Unies, ont "adopté" la déclaration universelle des droits de l'homme du 10 décembre 1948 (Art. 8) et le pacte international relatif aux droits civils et politiques du 16 décembre 1966

1 R. Cassin, Dualité de juridictions, la justice PUF, p. 720.

2 J. Riviero, Sanction juridictionnelle et règle de droit, Mélanges Julliot de la Morandiere, Dalloz 1964, p. 457. 
(art. 2-3). Au plan régional, la quasi totalité de ces Etats on ratifié la Charte Africaine des Droits de l'Homme et des Peuples du 28 juin 1981 (art. 7) ou y ont adhéré. Au plan national, les constitutions de ces Etats, tout particulièrement celles issues du mouvement récent de démocratisation, consacrent ce droit et les codes de procédure civile se chargent de leur mise en oeuvre. Il importe à ce stade de préciser que le jus en question est le droit moderne, c'est à dire le droit d'inspiration occidentale, qui s'exerce devant des juridictions d'Etat. La justice africaine rejoint en cela celle de l'Occident et souffre de ce fait presque des mêmes maux.

Mais le droit de recourir à la justice se trouve dans son exercise en situation concurrentielle voire conflictuelle permanente avec les droits traditionnels. Cette situation s'inscrit dans le contexte général de la survivance du conflit colonial qu'il importe de préciser.

Le droit actuellement en vigueur en Afrique, hérité du droit colonial, est un système plurijuridique composé à la fois du droit d'inspiration occidentale et des divers droits africains, y compris les droits islamisés. Pour marquer la distinction entre le droit d'inspiration occidentale d'une part et d'autre part tous les autres droits, on a pu qualifier ce système de dualiste. Cette qualification a certes le mérite de la simplification, mais ne rend pas compte de la réalité du droit en Afrique. En fait, le système colonial reconduit deux ordres juridiques auxquels il emprunte son caractère inégalitaire, mettant en opposition le droit d'inspiration métropolitaine, droit dominant et les droits africains, droits dominés. Ce conflit de type spécial, opposant les lois internes d'un même Etat, a été dénommé par R. Maunier "conflit colonial" ${ }^{3}$. Si, en raison de la souveraineté et la naturalisation, ce conflit perd sa dénomination coloniale, il se résoud, tout comme durant la période de domination, en principe aux dépens des droits africains qui demeurent une législation d'exception et au profit du droit occidental qui est imposé comme droit commun. Mais à l'envahissement du droit dominant étranger qui, ayant le support étatique, triomphe sur le plan juridique formel, les droits dominés qui, eux, bénéficient de l'appui populaire, opposent de vives résistances. ${ }^{4}$ Celles-ci constituent des obstacles spécifiques, propres à l'Afrique, susceptibles de paralyser l'accès aux juridictions d'Etat.

Il résulte de l'ensemble des données précédentes que les obstacles à l'accès à la justice tiennent à la fois aux textes et au contexte.

R. Maunier, Sociologie coloniale, introduction à l'étude du contact des races, Domat chrétien, Paris 1932, T2, p. 176.

4

Voir R. Degni-Segui, Codification et uniformisation du droit en Afrique, in Encyclopédie juridique, 1982, T1, l'Etat et le droit, p. 453. 


\section{Les obstacles tenant aux textes}

Les difficultés d'accès à la justice sont le fait de textes, s'analysant en des obstacles juridiques et surtout institutionnels. Mais ceux-ci, sans être l'apanage de l'Afrique, étant la rançon du mimétisme, y sont particulièrement difficiles à surmonter. Ils se ramènent à l'éloignement du justiciable, aux lenteurs de la procédure judiciaire et aux coûts du procès.

\section{A. L'éloignement du justiciable}

L'accessibilité à la justice est fonction de la proximité spatiale des juridictions. Des efforts ont été fournis dans ce sens visant à rapprocher la justice du justiciable, mais ils restent encore très limités.

\section{Des efforts pour rapprocher la justice du justiciable}

Ces efforts ont consisté à créer un grand nombre de juridictions. Ce sont d'abord les justices de paix créées dès après l'indépendance. Il en va ainsi, à titre d'exemple, du Congo (loi du 29 mai 1961) ou du Mali (loi du 15 mai 1961). Ce sont ensuite d'autres juridictions instituées dans certains Etats, souvent en remplacement des tribunaux de paix. Elles portent des dénominations diverses: tribunaux populaires locaux au Bénin, tribunaux populaires de conciliation au Burkina Faso, tribunaux populaires au Congo, tribunaux d'instance ou de lère instance et leurs sections détachées en Côte d'Ivoire...

Ces juridictions présentent un triple avantage se rapportant à leur nombre, leur ressort territorial et leurs compétences. Elles sont tout d'abord très nombreuses, l'objectif de la loi étant d'assurer la couverture judiciaire de tout le territoire national. Ainsi, le Rwanda peut-il déjà en 1979 s'énorgueillir de ses 119 tribunaux de Canton répartis sur une superficie de $27.000 \mathrm{~km}^{2}$, et le Burkina Faso de ses 8.000 tribunaux populaires de conciliation, auxquels il faut ajouter 300 tribunaux populaires départementaux ${ }^{6}$ pour une superficie de $274.122 \mathrm{~km}^{2}$. Elles sont ensuite circonscrites dans un ressort territorial tendant à réduire la distance qui sépare la justice du justiciable: ce sont les zones, secteurs ou quartiers des centres urbains ou des villages. Elles reçoivent enfin des compétences relative-

Filip Reyntjens, Assistance judiciaire et barreau en Af rique et au Rwanda en particulier, Penant, Janv. Fev. Mars 1979, p. 15.

Salif Yonaba, L'accès à la justice en droit Burkinabé, in Revue Burkinabé de droit, Janv. 1988, p. 155. 
ment limitées se rapportant notamment aux petits litiges, en matière civile et commerciale pour permettre leur solution rapide. La procédure y est d'ailleurs simplifiée.

\section{Les limites de la politique de rapprochement}

En dépit des efforts déployés, le juge reste encore, en Afrique, séparé du justiciable pour au moins trois raisons. La première, c'est que tous les Etats n'ont pas consenti à l'effort de décongestion de l'appareil judiciaire. On mentionnera quelques exemples: la Côte d'Ivoire ne dispose que de 3 Cours d'Appel, de 7 tribunaux de lère instance avec leurs 39 sections détachées $^{7}$, la Guinée de 2 Cours d'Appel et de 6 tribunaux, la République Centrafricaine de 16 tribunaux de Grande instance, de 52 tribunaux d'instance et le Tchad d'une Cour d'Appel de 50 juridictions de base. Si l'on osait une comparaison entre d'une part le Tchad avec ses 50 juridictions de base pour une superficie de $1.284 .000 \mathrm{~km}^{2}$ et d'autre part le Rwanda avec ses 119 tribunaux de base pour une superficie de $27.000 \mathrm{~km}^{2}$, l'on apprécierait davantage l'avance voire la performance du second autant que le retard du premier.

La seconde, c'est que la distance n'est pas dans tous les cas réduite et l'obstacle à l'accessibilité demeure, en raison soit de l'ineffectivité de l'institution, la juridiction étant créée mais non ouverte, ne fonctionne pas, soit de l'inégale répartition sur le territoire. L'exemple le plus typique nous est fourni par le Burkina Faso qui connaît une intense activité normative en la matière. En 1988 sur 30 tribunaux populaires de la révolution seuls 7 fonctionnaient. Le professeur Salif Yonaba qui rapporte l'exemple note que "cette remarque vaut également pour les autres tribunaux populaires" ${ }^{\prime 8}$. La troisième, c'est que certaines de ces juridictions dont les tribunaux populaires n'ont pas toujours de compétence juridictionnelle. Ce qui réduit d'autant la portée de l'oeuvre accomplie. Le Burkina Faso fournit là encore une illustration type avec les tribunaux populaires de conciliation qui, comme leur nom l'indique, se bornent à la conciliation et à connaître de petits litiges pour lesquels les tribunaux n'attirent guère les justiciables qui, le plus souvent, craignent les lenteurs de la justice.

\section{B. Les lenteurs de la procédure judiciaire}

Tout comme la réduction de l'espace, de la distance qui sépare la justice du justiciable, la réduction du temps, de la durée du procès constitue une préoccupation traditionnelle. Les lenteurs de la justice ne sont en effet rien d'autre que des dénis de justice qui amènent les

Ce chiffre n'a d'ailleurs été atteint que depuis le 15 mai 1992, cf. decret 92-135 portant création de la Cour d'appel de Daloa.

8

Salif Yonaba, op. cit., p. 176. 
justiciables à s'en défier. Il importe d'examiner comment se manifeste ce disfonctionnement avant d'en rechercher les causes.

\section{Les manifestations}

Même si la procédure judiciaire est relativement simplifiée devant les juridictions de base, elle demeure dans l'ensemble longue et lente surtout en parcourant tous les degrés de juridiction.

A la différence de la France, on ne dispose malheureusement pas de statistiques en la matière. ${ }^{9}$ Néanmoins ce qui paraît certain c'est que le traitement des procédures s'étale sur plusieurs mois voire plusieurs années. Et l'anomalie peut intervenir à n'importe quel stade de la procédure, de l'instruction à l'exécution de la décision en passant par le jugement ou affecter toute la procédure.

Ainsi, en Côte d'Ivoire, il est courant qu'une détention préventive dure 6, 8, voire 11 ans au lieu de 4 mois renouvellable, se transformant ainsi en détention définitive; ${ }^{10}$ les trois mois dans lesquels la Cour d'Appel doit statuer ne sont pas non plus respectés et les démocrates injustement incarcérés le 18 Février 1992 en ont été victimes. Déjà, le 13 Février 1987, le ministre de la justice, dans une circulaire portant instructions générales relatives au fonctionnement des juridictions, tout en reconnaissant les difficultés à respecter le délai, en stigmatisait l'excès en ces termes: "si dans la pratique il apparaît difficile de respecter ce délai, il me semble cependant qu'entre le délai légal et le délai qu'on constate il y a un écart excessif" ${ }^{11}$. Enfin, 8 mois ont été nécessaires pour délivrer la grosse d'une ordonnance de référé en expulsion de locataire. ${ }^{12}$ C'est ce qui explique que la circulaire précitée du ministre recommande aux magistrats de veiller "à la mise en état des minutes et à ce que les grosses et expéditions soient délivrées dans les délais les plus convenables".

Les mêmes errements se retrouvent au Burkina Faso où la durée des litiges s'étend sur plusieurs années atteignant, quelquefois, la décennie et avec une mention spéciale pour l'espèce Kabore Ali Noaga c/ Derme Moussa qui a franchi la barre des 25 années. $^{13}$ Le

9 Sur ce point voir J. Francis Overstake, Cours de droit judiciaire privé, 1992, pp. 96 et s.

10 Ligue ivoirienne des droits de l'Homme, Je connais mes droits, cahiers 2, Les libertés physiques, Avril, Mai, Juin 1993, p. 22.

11 Circulaire 001/MJ/Cab/1 du 13 Fév. 1987.

12 Rapporté par Kipre E. Baroan, Les entreprises et la Justice, in La Justice en Afrique, $\mathrm{n}^{\circ}$ Spécial Afrique Contemporaine, 1990, p. 84.

13

Rapporté par S. Yonaba, op. cit., p. 156. 
syndicat de la magistrature, à l'occasion d'un séminaire tenu en Août 1986, n'a pas manqué de stigmatiser le disfonctionnement de la justice en ces termes: "... Notre syndicat a toujours mis l'accent sur les lenteurs de la justice, la prolifération des lois de circonstance, l'usage abusif des voies de recours, les difficultés d'exécution des décisions de justice ..."14.

Les Cours Suprêmes, qui ne souffrent pourtant pas encore de l'encombrement que connaissent leurs homologues européennes, ne sont pas davantage à l'abri des procédures longues et lentes. ${ }^{15}$ C'est ainsi que les délais des litiges administratifs atteignent facilement les huit ans en Côte d'Ivoire ${ }^{16}$ et les dix ans au Bénin ${ }^{17}$. Au Gabon, en revanche, avec un peu plus d'affaires, le délai oscille entre 4,5 et 18 mois avec une moyenne de 12 mois $^{18}$

\section{Les causes}

Les causes du disfonctionnement de l'appareil judiciaire sont multiples et diverses. On peut en retenir principalement trois.

Certaines sont internes au fonctionnement du service public de la justice. Elles se rapportent d'abord: aux procédures demandant de longues mesures d'information, surtout lorsque les dossiers sont traités successivement par plusieurs magistrats à l'instruction et au parquet; aux nombreux renvois d'une audience à une autre; aux retards dans la transmission du dossier d'une juridiction à une autre, au non respect des délais légaux; à la non-comparution des témoins... Elles se rapportent ensuite aux conditions de travail des magistrats: ceux-ci sont en nombre insuffisant et exécutent le service public dans des conditions matérielles peu propices à l'accomplissement de leur noble mission et surtout la documentation juridique de base fait souvent cruellement défaut.

D'autres sont le fait des magistrats eux-mêmes qui adoptent des comportements emprunts du laxisme habituel des fonctionnaires africains. La circulaire précitée de 1987 du minis-

ibidem, p. 147.

15 Voir sur ces points Les Cours Suprêmes en Afrique, sous la direction de G. Conac et de J.D. Gaudusson, Université de Paris, Economica, Paris 1988, 4 tomes.

C.S.C.A. 20 Mars 1968, Etat de CI c/ Groupement Français d'assurances, Bull. C.S. 1969, nº Spécial J. 16; C.S.C.A. 14 Janvier 1970, Société des Centaures Routiers, R.I.D. $1971 \mathrm{n}^{\circ} 1$, pp. 11 et s.; A.J.D.A. 1970, pp. 560 et s.

17 Voir Th. Holo, Contribution d'études des chambres administratives, cas de la chambre administrative de la Cour populaire centrale du Bénin, in Les Cours Suprêmes, op. cit., p. 26.

18

Voir Yves Luchaire, La chambre administrative et la Cour Suprême du Gabon, in Les Cours Suprêmes, op. cit., pp. 202 et s. 
tre ivoirien de la justice dénonce cette situation. Estimant que, pour l'ensemble des cabinets pendant deux années judiciaires (1985-1986), la moyenne mensuelle de 9 dossiers à Abidjan et de 8 à Bouaké n'est pas le fait du volume des informations ouvertes dans les parquets, le ministre en impute la responsabilité aux magistrats. Il leur est en outre reproché: la non tenue d'audiences sans motifs sérieux (cas de force majeure); les mauvaises habitudes consistant à "n'enroler que certaines affaires" et 'a renvoyer d'autres aux calendres grecques. La dernière habitude consiste à ruser pour faire traîner les affaires au préjudice du justiciable. Le ministre rapporte en effet, que les magistrats ivoiriens on pris "la mauvaise habitude consistant à renvoyer dès le mois d'Avril les affaires aux dernières audiences du mois de Juillet, précédant les vacations judiciaires, puis aux mois d'Octobre et Novembre pour être enfin renvoyées aux mois de Décembre, Janvier et Février". Le ministre déplore par ailleurs qu'"il n'est pas convenable que les affaires soient mises en délibéré pour la même période de Juillet sachant pertinemment que ces délibérés ne seront pas vidés mais rabattus".

D'autres encore sont le fait des pouvoirs publics, tout particulièrement du ministre de la justice, qui procèdent à des mutations et déplacements fréquents des magistrats et auxiliaires de justice. Ces mutations et déplacements opérés souvent pour des raisons politiques compromettent le fonctionnement régulier du service public de la justice en aggravant les maux habituels qui minent ledit service, allant jusqu'à la perte des dossiers L'usager qui est ainsi victime d'un véritable déni de justice peut-il avoir la patience nécessaire et suffisante pour pursuivre la procédure, surtout lorsqu'il envisage son coût?

\section{Le coût du procès}

Le coût de la justice constitue le troisième obstacle traditionnel qui en entrave l'accès. La gratuité de la justice, du reste très mal comprise des Africains, perd tout sens devant les frais élevés de l'instance que l'assistance judiciaire limitée ne peut compenser.

\section{Des frais élevés}

Le "drame" pour le justiciable africain, c'est que non seulement les frais de l'instance restent très élevés mais encore et surtout la charge d'un procès reste difficile à évaluer.

L'instance est l'occasion pour le justiciable d'exposer de multiples frais, appelés "frais" ou "dépenses". Les codes de procédure civile dressent une liste des dépenses qui comprennent: un certain nombre de droits, taxes et redevances; les frais de transports des magistrats et autres en cas de descente sur les lieux; les indemnités dues aux témoins en cas 
d'enquêtes; les rémunérations des experts; les émoluments des avocats, huissiers et autres. 19

Sans entrer dans les détails de la liste longue et non exhaustive, il importe de relever que les justiciables africains dans leur écrasante majorité ont des revenus dérisoires voire insignifiants, de sorte que les différents actes successifs occasionnant des frais peuvent respectivement les dissuader d'engager ou de poursuivre l'action en justice. Il en va ainsi de l'assignation, exploit d'huissier destiné à signifier les actes de procédure ou de la consignation entre les mains du greffier de la somme nécessaire au paiement des frais. Il en va de même et surtout de la constitution d'avocat qui, sans être dans tous les cas obligatoire, paraît nécessaire en raison des subtilités voire des pièges de la procédure et revient très chère. Il en va de même encore de l'issue du procès. Si le plaideur "succombe", c'està-dire s'il est perdant, il supportera toutes les dépenses. Et, s'il est gagnant, il ne sera pas pour autant à l'abri de certaines dépenses dont les honoraires de l'avocat et les voyages et déplacements effectués (sauf exceptionnellement décisions contraires conventionnelles ou juridictionnelles). Pour couronner le tout, il peut, soit manquer de ressources pour faire exécuter la décision judiciaire afin d'obtenir le remboursement des dépenses et éventuellement de sa créance, soit se heurter à l'insolvabilité du débiteur.

Mais, au delà de la multiplicité des frais et du coût de la justice qui en résulte, ce qui inspire davantage la crainte du justiciable, c'est la difficulté à évaluer la charge d'un procès. ${ }^{20} \mathrm{M}$. André Rials insiste sur ce point en relevant les différents paramètres variables qui entrent en ligne de compte. Au nombre de quatre, ils se présentent ainsi: l'issue aléatoire de beaucoup d'affaires notamment en raison des interprétations différentes des textes fournis par les tribunaux; la durée incertaine du procès qui peut s'arrêter en première instance ou au contraire franchir tous les dégrés de juridiction jusqu'en cassation; en cas de triomphe le non-remboursement des frais exposés en raison de l'insolvabilité du perdant; enfin, l'impossibilité de déterminer avec précision les frais et honoraires de l'avocat en conséquence de ce qui précède. L'auteur conclut en ces termes: "la charge financière peut ainsi varier du simple au décuple et représenter parfois une dépense très supérieure à l'intérêt du litige" ${ }^{21}$ Ce qui est vrai du justiciable français l'est a fortiori du justiciable africain qui manque de ressources. Aussi se voit-il contraint de recourir à l'assistance judiciaire.

Voir sur ce point Gilbert Mangin, Emile Mamy et Antoine Rubbens, Les frais de l'instance, in Encyclopédie juridique de l'Afrique, T4 Organisation judiciaire, procédures et voies d'exécutions, NEA 1982, pp. 217 et $\mathrm{s}$.

Voir sur ce point $A$. Rials, L'accès à la justice - Que sais-je?, N²735, PUF, Paris, 1993, p. 11. Voir également "Le coût d'un procès" in 50 millions de consommateurs, 21 nov. 1991, rapporté par A. Rials. ibidem, op. cit., p. 12. 


\section{Une assistance judiciaire limitée}

L'assistance judiciaire vient heureusement faciliter l'accès du prétoire à des justiciables dont les revenus sont insuffisants. Introduite en Afrique noire francophone par le législateur colonial (lois des 11 Janvier 1851 et 10 Juillet 1910), l'institution a été reprise par la plupart des codes de procédure civile. Elle repose sur le principe de l'égalité de tous devant la loi, plus spécifiquement devant la justice. Le justiciable qui n'a pas les moyens de supporter les charges d'un procès se voit ainsi ouvrir le prétoire pour faire valoir ou pour défendre, sans frais, ses droits contestés au même titre ou contre un autre citoyen qui dispose de ressources suffisantes.

L'assistance judiciaire, telle que réglementée par le législateur africain, paraît faciliter l'exercice effectif de l'action en justice pour trois raisons bien simples. La première, c'est que les conditions d'attribution ne sont pas exigentes, la condition essentielle se ramenant à l'"indigence" ou à l'"insuffisance" des ressources du candidat. Certains codes exigent en outre que la cause présente des chances de succès. La seconde, c'est que la procédure d'attribution est relativement simplifiée: l'assistance est accordée par un bureau à la suite d'une requête qui peut être introduite à tous les stades de la procédure. La troisième, c'est que la portée de l'assistance est relativement étendue: l'aide est totale, quelquefois partielle, déterminée en fonctions des ressources ${ }^{22}$ et concerne toutes les procédures contentieuses autant que gracieuses.

L'assistance judiciaire comporte cependant deux limites importantes qui en réduisent considérablement la portée, les unes de droit, les autres de fait. Les premières ont trait aux insuffisances de la réglementation. Les critères d'attribution som imprécis, laissant une marge de discrétion assez large au bureau de l'assistance judiciaire qui peut en abuser en introduisant du favoritisme et fermer le prétoire à de vrais indigents au profit de faux. Quel est le seuil de l'insuffisance? Peut-on apprécier les chances de succès d'une cause? Ces imprécisions sont d'autant plus à redouter que certaines réglementations telles que celles de la Côte d'Ivoire prescrivent que les décisions du bureau de l'assistance judiciaire "ne sont susceptibles d'aucun recours de la part des parties" ${ }^{23}$. Par ailleurs, l'assistance judiciaire se limite à l'aide juridictionnelle à l'exclusion d'une aide à l'accès au droit comme c'est le cas en France depuis 1992, alors que l'Afrique, semble t-il, en a autant besoin sinon plus que la France ${ }^{24}$. Les secondes se rapportent à la portée pratique de

22

23

24

Voir G. Mangin et autres, op. cit., p. 220.

Art. 13 decret $n^{\circ} 75-319$ du 9 mai 1975 fixant les modalités d'application de la loi $n^{\circ} 72-833$ du 21 déc. 1972 portant Code de procédure civile, commerciale et administrative en ce qui concerne l'assistance judiciaire.

Voir loi française $n^{c}$ 91-647 du 10 juillet 1991 relative à l'aide juridique qui est entrée en vigueur le ler Janv. 1992. 
l'institution. Celle-ci est, faute d'information, limitée dans son application au petit nombre d'indigents des centres urbains, à l'exclusion de la grande masse des justiciables des villages et même des villes. De plus, ce petit nombre d'indigents risque de recevoir une assistance judiciaire très limitée autant financièrement que juridiquement. Financièrement, l'indigent sera amené, avant même de recevoir l'assistance, à exposer certains frais, soit pout solliciter des pièces, soit pour faire accélérer la procédure d'attribution ou même simplement pour en bénéficier effectivement: corruption oblige. En Côte d'Ivoire c'est le "fais-nous fais", et au Zaïre M. Mubiala rapporte que "l'indigent se trouve obligé de débourser une importante somme d'argent pour avoir l'attestation d'indigence" ${ }^{25}$. De plus, lorsque l'assistance est partielle, il se voit contraint d'assurer une partie des frais d'instance. Juridiquement, la désignation d'office des avocats et la "très lourde charge" ${ }^{26}$ que l'assistance judiciaire fait peser sur eux peuvent affecter substantiellement la qualité de la prestation fournie. Il est, en effet, à craindre que l'avocat soit peu motivé, préférant consacrer tout son art aux grosses affaires payantes au préjudice de celles de l'indigent et ou l'abandonner purement et simplement entre les mains de stagiaires inexpérimentés. La nature humaine risque ainsi de prendre le pas sur l'éthique professionnelle ${ }^{27}$. La victime de ce choix c'est l'indigent qui peut perdre son procès ou renoncer à l'exercice de son action s'il garde à l'esprit les obstacles liés au contexte.

\section{Les obstacles tenant au contexte}

A la différence des obstacles précédents, ceux-ci sont spécifiques à l'Afrique. Ils relèvent d'ordres divers: politiques, économiques, sociologiques, psychologiques, culturels etc... On peut les regrouper en deux catégories, tenant les unes au système politique et les autres à la méconnaissance du droit extranéen.

\section{A. Le système politique af ricain}

Cette barrière paralysant l'exercice du droit de recourir aux juges est le fait des pouvoirs publics dans une Afrique dont le système politique se caractérise par l'autoritarisme, la justice liée et l'ineffectivité institutionnelle.

Mubiala, L'individu devant la justice au Zaïre: de l'arbre à palabre aux cours et tribunaux, Penant mai-sept. 1992, p. 192. 


\section{L'autoritarisme}

Le système politique africain est développementaliste et autoritaire. L'idée maîtresse c'est que l'unité et la construction nationales imposent un pouvoir fort et autoritaire qui prend souvent l'allure de dictatures sanglantes. ${ }^{28}$ Il en résulte pour le justiciable la crainte de représailles qui le conduit à s'abstenir de traîner les autorités administratives devant les tribunaux et à rechercher des voies négociées de règlement des conflits.

La crainte de représailles procède de l'opinion très répandue en Afrique suivant laquelle attraire l'administration devant les tribunaux relève de la témérité voire de l'inconsistence, l'entreprise étant très risquée. Le ministre centrafricain de la justice, en 1969, s'en faisait ainsi l'écho: "on ne peut dire qu'en République Centrafricaine le recours aux juridictions administratives soit encore véritablement entré dans les moeurs. Quereller l'Administration devant un juge paraît encore une entreprise téméraire voire incivique" ${ }^{29}$. Mais la crainte de représailles s'exprime surtout à l'égard des recours portés devant les hautes juridictions administratives contre les actes des autorités administratives, tout particulièrement ceux du chef de l'Etat. L'Africain croit, en effet, qu'attaquer une décision présidentielle c'est attaquer le Président de la République et, au déla de cette haute autorité politique et administrative, sa personne. C'est sans doute cette peur qui explique la rareté de tels recours et qui a conduit, en Côte d'Ivoire, le sieur Akpa Akpro à déférer à la censure de la Cour Suprême non le décret présidentiel qui le mettait en position de réformé, mais plutôt et curieusement le refus du ministre de la défense de rapporter ledit décret. $^{30}$ Le juge a déclaré ce recours biaisé irrecevable, au motif que l'arrêté querellé ne modifiait pas l'ordonnancement juridique. Mais si le recours avait été directement dirigé contre le décret présidentiel, le juge aurait-il eu l'audace de le déclarer recevable et d'en prononcer l'annulation? Rien n'est moins sûr, risquant de s'exposer aux "foudres" du pouvoir. ${ }^{31}$ Au Tchad, la chambre administrative et financière de la Cour d'Appel n'a pris aucun risque. Saisie de la "seule requête" en contestation des mesures prises par le Président Tombalbaye en 1973, elle l'a bloquée et ne s'est prononcée qu'après la mort du "tyran". ${ }^{32}$ Le juge suprême beninois fait en revanche preuve de plus de courage et

Voir sur ce point les dictatures sanglantes de Bokassa en Centrafrique, Macias N'Guema en Guinée Equatoriale, Idi Amin Dada en Ouganda, qui ont été renversées en 1979, sans oublier celles de Mobutu au Zaïre, d'Eyadema au Togo etc...

R.J.P.I.C. 1969, n ${ }^{\circ}$ 4, p. 935, rapporté par P.F. Gonidec, La place des juridictions dans l'appareil d'Etat, in Encyclopédie Juridique de l'Afrique, Tl, l'Etat et le droit, p. 235.

Cour Suprême, chambre administrative, 22 Juillet 1981, Akpa Akpro c/ ministre de la défense (inédit).

Voir sur ce point $F$. Wodie, La législation, in Encyclopédie juridique de l'Afrique, T1, l'Etat et le droit, p. 327.

32

Rapporté par A. Dadi et A. Moyrand, La juridiction administrative du Tchad, in Les Cours Suprêmes en Afrique, T3, pp. 303-304. 
d'audace, ayant annulé successivement et dans un intervalle de 12 années deux décisions individuelles du Président de la République, reprochant à la première, qui revoquait un fonctionnaire, d'être entachée d'erreur matérielle et à la seconde, qui portait un ordre de réquisition, d'avoir violé la loi. ${ }^{33}$ Si ces décisions juridictionnelles témoignent du courage du juge autant que de la témérité des justiciables qui l'ont provoquées, elles font partie des rares exceptions qui confirment la règle.

L'Africain recherche, en effet, presque toujours, une voie négociée de règlement des conflits $^{34}$, en pareille occurence. Il peut gravir tous les échelons administratifs, du Préfet au Président de la République en passant par le ministre à la quête d'une solution négociée de son problème. Mais le plus souvent, il préfère avoir affaire à Dieu qu'à ses Saints, en saisissant directement le chef de l'Etat pour solliciter de lui qu'il entende sa cause en se fondant sur la Justice et l'équité et non sur la rigueur des textes. Le recours systématique à l'arbitrage du Président de la République se trouve justifié par sa triple qualité de chef suprême de l'administration, président du Conseil Supérieur de la magistrature, premier magistrat de l'Etat. C'est dans ce contexte qu'il convient d'inscrire le "dialogue à l'ivoirienne" qui n'est pas le propre de la Côte d'Ivoire mais est largement utilisé dans presque tous les Etats africains notamment au Togo, au Mali, au Zaïre ${ }^{35}$, au Bénin ${ }^{36}$, au $\operatorname{Tchad}^{37} \ldots$ de sorte qu'il serait plus exact de parler de "dialogue à l'africaine". Celui-ci se trouve d'ailleurs institutionnalisé dans le recours administratif préalable au recours juridictionnel de l'annulation que la plupart des législations rendent obligatoire en exigeant du justiciable qu'il provoque une décision administrative supplémentaire (le rejet), alors qu'il dispose déjà d'une décision attaquable. Le législateur, fait-on remarquer, veut voir épuiser les moyens du dialogue. ${ }^{38}$ Cette remarque paraît fondée, car on ne comprendrait pas autrement le caractère d'un moyen d'ordre public, susceptible d'être soulevé d'office par le juge. Les justiciables congolais, rapporte J.M. Bretton, bien que leur droit ait opté pour une solution différente qui ne fait pas du recours préalable une formalité obligatoire devant le juge de l'excès de pouvoir, sont pour la plupart "... naturellement plus enclins, en toutes circonstances, à saisir dans un premier temps l'Administration, et à rechercher le règlement direct de leurs litiges avec elle, par le biais d'un recours qui se présente comme préalable de conciliation, en raison probablement d'une crédibilité plus limitée dans

Première décision A. Behanzin, 14 Avril 1962, et deuxième décision Edmond Alapini, 19 Juillet 1974, rapporté par Th. Holo, op. cit., pp. 25-26.

$E$. Leroy, Le justiciable africain et la redécouverte d'une voie négociée de règlement des conflits, in La Justice en Afrique, p. 111.

35

Cf. E. Leroy, op. cit., p. 113.

Cf. Th. Hollo, op. cit., p. 26.

Cf. A. Dadi et A. Moyrand, op. cit., p. 299.

38

Voir Yves Daudet, Chronique jurisprudentielle, in R.I.D. 1976, pp. 1-2-49. 
l'intervention d'un juge dont le rôle est trop mal connu" ${ }^{39}$. La crédibilité du juge est encore plus affectée par le caractère lié de la justice.

\section{La justice liée}

A la différence de l'obstacle précédent qui ne bloque que l'accès de la "justice administrative" en tant qu'elle veut mettre en cause l'administration elle-même, cette barrière détourne le justiciable de toutes les juridictions. C'est en effet la justice elle-même qui perd toute crédibilité aux yeux des populations africaines du fait de sa partialité et de sa vulnérabilité, étant soumise aux pressions de toutes sortes, tout particulièrement celles du pouvoir et de l'argent.

La politisation de la justice ${ }^{40}$ procède elle-même de la confusion totale des pouvoirs qui, en dépit de la constitutionnalisation de l'indépendance de l'autorité judiciaire, lie celle-ci au pouvoir exécutif détenu par le chef de l'Etat. J.P. Passeron notait, en 1966 en ce sens: "en définitive la justice en Afrique Noire reste et restera marquée par le régime d'unité du pouvoir qui confie le rôle essentiel au chef de l'Etat, érigé en véritable guide de la Nation, animant un parti unique ou dominant, majoritaire à l'Assemblée et pouvant éliminer sans rencontrer d'obstacles toute opposition susceptible de nuire à la cohésion nationale, au développement du pays" ${ }^{41}$. Cette remarque, qui demeure d'actualité, en dépit du processus de démocratisation, est confirmée par le président zaïrois Mobutu qui relevait en mai 1989, au sommet de la Francophonie à Dakar que "le chef africain ne partage pas son pouvoir" ${ }^{42}$. Un tel pouvoir concentré, qui appartient en propre au "Chef" ne peut que s'aliéner le pouvoir législatif et surtout le pouvoir judiciaire qui sera amené à condamner sans preuve des citoyens, particulièrement les opposants au régime et, en cas de résistance du juge, le sanctionner, lorsqu'il n'annule pas purement et simplement sa décision.

La Côte d'Ivoire recèle de larges ressources en la matière. Trois affaires peuvent être citées. Dans la première, l'espèce Gnadre Teti et autres, le Président de la République, se prenant pour un degré supérieur de juridiction, décide "... en dernier ressort ... de limiter les effets de l'arrêt de la Cour Suprême (chambre administrative) aux seuls candidats malheureux ...". L'arrêt de la Cour Suprême daté du 8 Février 1985 annulait les examens

J.M. Bretton, La jurisprudence administrative au Congo, in Les Cours Suprêmes en Afrique, T3, op. cit., p. 98.

41 J.P. Passeron, Le pouvoir et la justice en Afrique noire francophone et à Madagascar, Paris, Pédone, 1966, pp. 157-158.

42 Rapporté par A. Bourgi et Ch. Casteron, Le printemps de l'Afrique, Hachette 1991, p. 30. 
du Certificat d'aptitude à la profession d'avocat (C.A.P.A.). ${ }^{43}$ Dans la seconde, Ministère Public c/ Gbagbo Laurent, Degni-Segui René et autres, la Cour d'Appel qui s'apprêtait à relaxer le 23 juin 1992 des prévenus condamnés par le tribunal correctionnel d'Abidjan le 6 mars 1992 sans aucune preuve, est sommée de proroger son délibéré d'une semaine. Et, le 26 juin, contre toute attente, elle est dessaisie par ordre du procureur général sur instruction du Ministre de la Justice. Le dessaisissement de la Cour se fondait sur la "suspiscion légitime" de son président, accusé d'être un "démocrate" au même titre que les prévenus. $^{44}$ Le 24 Juillet 1992, sur "injonction du Président de la République, l'Assemblée Nationale, qui auparavant se refusait à toute intervention, nonobstant l'arrestation arbitraire de quatre de ses membres, adoptait à l'unanimité une loi d'amnistie pour libérer les détenus. Le président de la Cour d'Appel est relevé de ses fonctions dès la rentrée judiciaire d'octobre 1992. Dans la troisième affaire, Essoh Lath, la même Assemblée à qui il était demandé de lever l'immunité parlementaire d'un député de la majorité au pouvoir, soupçonné d'avoir assassiné sa concubine, refuse en raison des liens de parenté existant entre le de cujus et le ministre de la Justice. C'est ce qu'expriment le 20 Juillet 1993 à l'Assemblée Nationale certains membres du parlement en ces termes: "l'environnement social et judiciaire n'est pas du tout favorable au député de Port-Bouët pour des raisons de filiation entre la victime, l'ex-épouse du député Essoh Lath et le garde des Sceaux, ministre de Justice" ${ }^{45}$. L'un d'entre eux, ancien ministre de la Justice, déclare sans détours le 28 Juillet 1993: "c'est envoyer un député à l'abattoir que de laisser la justice mener son cours" $^{\prime \prime 6}$.

La pression de l'argent n'est pas moins forte et revêt une portée beaucoup plus générale, affectant la justice ordinaire par opposition à la justice politique, encore que la distinction entre les deux ne soit pas bien nette. Elle s'inscrit dans le contexte global de la corruption généralisée qui gangrène toute d'Administration africaine. "La politique du ventre", note Marc Debene, est aussi une "attitude judiciaire" ${ }^{47}$. Le recours aux épices conduit les magistrats à rançonner les plaideurs et à vendre leurs services aux plus offrants. Les auxiliaires et plus particulièrement les avocats ne sont pas en reste. Plutôt que de se donner la peine de plaider en droit, une partie de ceux-ci, fait remarquer Maurice Kamto, "préfère monnayer le procès aux frais du client" ${ }^{48}$. Une telle justice est loin d'être égale pour tous, établissant une discrimination à l'avantage des plus fortunés et au préjudice des

43

44

45

46

47

48

Voir René Degni-Segui, Droit administratif général, Abidjan 1990, p. 458.

Il s'agit de dirigeants et militants d'associations humanitaires, syndicales et politiques qui ont participé le 18 Fév. 1992 à une marche pacifique de protestation contre le refus du Chef de l'Etat de sanctionner les responsables d'une expédition punitive dans un campus universitaire.

Cf. journal La Voie n ${ }^{0} 551$ du 22 Juillet 1993, p. 3.

Cf. également journal La Voie ${ }^{\circ} 569$ du 12 Août 1993, p. 2.

Marc Debene, La justice sans juge, hier à demain, in La Justice en Afrique, op. cit., p. 90.

Maurice Kamto, Une justice entre tradition et modernité, in La Justice en Afrique, op. cit., p. 63. 
moins nantis qui représentent la plus grande partie de la population africaine et aux revenus dérisoires voire inexistants. Ainsi la célèbre phrase de Pascal aura survécu au temps: "selon que vous serez puissant ou misérable, les jugements de la Cour rendront blanc ou noir". L'on comprend dès lors la crainte légitime et la réserve des "misérables" africains à s'engager dans une aventure dont l'issue est incertaine, le procès étant perdu d'avance. L'on comprend également leur défiance à l'égard d'une justice monnayable donc dévoyée en contradiction flagrante avec leur propre conception de la justice.

\section{L'ineffectivité institutionnelle}

La situation du droit à la justice se complique davantage avec l'ineffectivité institutionnelle qui caractérise les régimes africains.

Les régimes militaires en donnent la meilleure illustration. Les coups d'Etat militaires ont, en effet, pour conséquence d'abroger ou de suspendre les textes de lois, dont la constitution. Il s'ensuit un vide juridique et institutionnel qui ôte aux citoyens les garanties à eux offertes pour assurer l'exercice de leurs droits élémentaires et des libertés fondamentales. Ce fut notamment le cas du Togo de 1967 à 1979, du Bénin de 1968 à 1979 ou encore de la Guinée de 1984 à au moins 1990, du Tchad de 1979 à 1982... L'ineffectivité institutionnelle a d'autant plus sévi que l'Afrique a connu en trente ans, c'est à dire de 1960 à 1990, plus de quatre vingt coups d'Etat soit une moyenne annuelle de trois. ${ }^{49}$ Au Bénin, par exemple, la Cour Suprême, particulièrement la chambre administrative, a connu une paralysie dans la période de 1965 à $1967 .^{50}$ De même au Tchad plusieurs périodes d'instabilité constitutionnelle, dont particulièrement celles allant de 1979 à 1982, ont paralysé le fonctionnement de la justice. ${ }^{51}$

L'ineffectivité institutionnelle n'est pas le propre des seuls régimes militaires; elle caractérise l'Afrique tout entière qui emprunte des institutions modernes à l'Occident pour les reléguer au musée des institutions juridiques. Il n'est que de rappeler ici que la démocratie, le multipartisme et les droits de l'homme, quoique consacrés par les différentes constitutions qui se sont succédées, n'ont pu avoir un début d'application qu'en 1990, soit trente ans après et, ce, à la faveur du mouvement de démocratisation.

L'explication que les chefs d'Etat donnent du décalage constant qui sépare le droit de la pratique, c'est que le droit dit moderne est investi d'une double mission d'unité nationale et

49

Rapporté par A. Bourgi, Ch. Casteran, op. cit., p. 115.

50 Voir Th. Hollo, op. cit., p. 28.

51

Rapporté par A. Dadi et A. Moyrand, op. cit., p. 305. 
de développement économique. Le Président ivoirien, se faisant le porte parole des Etats africains, déclare le 15 Août 1967 à Montréal: "dans nos pays la tâche la plus importante des gouvernants et d'introduire les structures, de promulguer les textes, de créer les organismes qui devancent le degré d'évolution de la société". Répliquant à l'objection du décalage qui s'établirait ainsi entre le droit et la réalité sociale, le Chef de l'Etat soutient que "l'écart entre la réalité d'aujourd'hui et les actes du législateur indique la direction dans laquelle doit s'engager l'effort de tous" ${ }^{\prime 52}$. La thèse du "droit du développement" qui attribue au droit occidental des vertus de développement économique et de progrès social n'est-elle pas un mythe? Un droit quelle que soit sa perfection technique formelle, doit être en relation avec la société et l'exprimer et non être un idéal, incompréhensible pour ceux qu'il doit régir et qui sont censés ne pas l'ignorer. ${ }^{53}$ A la vérité il ne s'agit que de démocratie et de liberté de façade à usage externe. M. Mendoza s'exclame: "quelle contradiction!" puis il déplore: "L'apparente dichotomie se résoud de façon élémentaire: une apparence sur le plan formel à usage externe et une réalité interne tragique" ${ }^{54}$. Celleci se traduit par la méconnaissance du droit extranéen.

\section{B. La méconnaissance du droit extranéen}

La méconnaissance du droit extranéen, c'est à dire du droit étranger, transplanté en Afrique, revêt en fait un double aspect: son ignorance par l'Africain et le refus de celui-ci d'y recourir, tout particulièrement de saisir les juridictions d'Etat.

\section{L'ignorance du droit}

L'un des obstacles majeurs à l'accès du prétoire en Afrique c'est l'ignorance du droit en général et de leurs droits en particulier par les citoyens qui sont pourtant censés ne pas les ignorer, en vertu de l'adage nemo legen ignorare censetur.

On peut se faire une idée approximative des difficultés d'accès au droit si l'on ne perd pas de vue que l'Afrique compte encore plus de $80 \%$ d'analphabètes ou de personnes très peu scolarisées. L'analphabétisme constitue pour cette masse de justiciables un handicap si sérieux qu'on est en droit de se demander si la présomption de connaissance de la loi, qui

52

Conférence prononcée sur le thème "unité et développement", in recueil des textes et documents du ministère de la Fonction Publique, imprimerie nationale 1974, p. 17.

53

R. Degni-Segui, Codification et uniformisation du droit, op. cit., p. 460.

54 E. Mendoza, L'immunité: une politique qui permet de se soustraire aux exigences législatives nationales et internationales, in Colloque international Démocratie, Développement, Droit de l'Homme, Torture, Manille 1991, p. 76. 
est une condition de son opposabilité, leur est applicable et si l'on ne devrait pas au contraire la renverser pour eux en posant: nul n'est censé connaître la loi. Mais, faute de pousser la logique à son terme, le législateur ne pourrait-il pas la retenir à mi-chemin en reconnaissant aux analphabètes un statut particulier d'incapacité juridique ${ }^{55}$ ? Les autres citoyens, c'est à dire les $20 \%$ restant, ne sont pas, pour la plupart, particulièrement attirés par le droit qu'ils considèrent comme une matière rébarbative et complexe dont l'accès n'est réservé qu'aux seuls initiés que sont les juristes.

Ils n'ont pas tort. Qu'il suffise en effet de relever en ce sens: l'incompréhension des textes légaux, la multiplicité et la complexité des conditions de recevabilité des recours contentieux (notion d'intérêt à agir, d'acte administratif), la brièveté des délais (délais très limités du recours pour excès de pouvoir généralement de deux mois comme en France), la complexité des procédures juridictionnelles longues et lentes, dont l'issue reste, de surcroît, incertaine... Autant de difficultés qui restreignent sinon paralysent l'accès au droit. Les praticiens du droit, eux-mêmes, avocats et juges, ne font pas exception à la règle, laissant souvent transparaître leur ignorance du droit public et soumettant la puissance publique au droit privé qui relève de leur formation et de leur spécialité. ${ }^{56}$ Désabusées, les populations rurales lancent: "les lois sont faites pour les gens de la ville et non pour les villageois" 57 et celles des centres urbains renvoient la balle "aux blancs", bien qu'elles-mêmes soient assimilées aux blancs. Cette situation est d'autant plus déplorable que l'information des citoyens sur leurs droits par l'Etat fait cruellement défaut, à tout le moins n'est pas organisé dans beaucoup de pays et que ses agents, particulièrement les forces de l'ordre, profitent de l'ignorance des Africains pour se livrer, en toute impunité, à des excès de pouvoir. Pour y remédier M. Adama Dieng, Secrétaire Général de la Commission Internationale des Juristes propose: "des projets d'assistance juridique pour les populations démunies du monde rural. Ces projets comportent notamment la formation et l'utilisation de para-juristes sur le terrain" ${ }^{58}$. Mais cette aide juridique à l'accès au droit entamera-t-elle leur détermination à refuser de recourir au juge?

Voir dans ce sens Yolande Tano, L'inaptitude juridique de l'analphabète, in Studi Giuridici ItaloIvoriani, Macerata 21-23 mars 1991, Ahi del convegno, Milano, Dote a Guiffre editore, 1992, p. 253. Voir dans ce sens $R$. Degni-Segui, Le droit de la responsabilité extracontractuelle de l'administration en Côte d'Ivoire, in Studi Giuridici..., op. cit., p. 79, et Bibombe-Muamba, Contentieux administratif au Zaïre in Les Cours Suprêmes..., T3, op. cit., p. 379. 


\section{Le refus de recourir aux juridictions d'Etat}

Les populations africaines vivent en marge du droit "moderne" et conformément à leurs coutumes respectives. Les codes civils ou successoraux continuent d'être boudés au profit des coutumes locales, telles que celles qui régissent le mariage, la dot, la polygamie, les successions, l'hospitalité... La même attitude est observée à l'égard des institutions et procédures "modernes" dont les juridictions.

Malgré les efforts déployés d'abord par l'Etat colonial et ensuite par l'Etat africain luimême pour "rapprocher la justice des justiciables", les "palabres" africains sont réglées en dehors des tribunaux et conformément à la coutume. Lors du premier congrès de l'Institut International du droit d'expression française tenu à Fort-Lamy en 1966, il fut stigmatisé la "fuite" des Africains devant les tribunaux coutumiers. Au second congrès, tenu l'année suivante à Dakar, on fit le même constat. Le l'avis du professeur Alliot "il n'y a pas plus de $1 \%$ des affaires coutumières qui sont soumises à la justice des tribunaux traditionnels, le reste étant règlé par les chefs selon leurs procédés ancestraux en dehors de toute intervention d'Etat" ${ }^{59}$. Sans pouvoir avancer de chiffres l'on peut af firmer que le même constat d'échec ne fait presqu'aucun doute. "Aujourd'hui", écrit Marc Debene, "un audit des systèmes judiciaires se solderait certainement par un résultat négatif" ${ }^{60}$. Et pour cause, le juge Keba M'Baye précise à propos du Sénégal: "la fierté du paysan sénégalais est de pouvoir dire: je n'ai jamais mis les pieds dans un tribunal ou un commissariat de police" $^{\prime 61}$. D'avoir affaire à la justice reste encore perçue presque partout en Afrique comme une atteinte à l'honorabilité et à la dignité humaine. L'Africain, même s'il vit dans une case ne supporte pas d'être enfermé dans une cage. Et, malheur au justiciable qui osera se prévaloir de sa décision contre sa communauté et même contre un des membres de celle-ci. Il s'y exclurait de lui-même ou s'exposerait à des sanctions. G. Pontie et M. Pilon expliquent: "La femme qui aura obtenu le divorce continuera à faire l'objet de pressions de la part de sa famille. Le mari délaissé qui n'aurait pas obtenu, au tribunal, le remboursement intégral des prestations liées au mariage, continuera à réclamer. Le dissident devra se résigner à quitter le village ou à payer sa réintégration sociale de quelques concessions ..."

L'indifférence voire l'hostilité des Africains à l'égard des institutions d'Etat ne surprend guère quand on sait qu'elles sont taxées d'institutions importées, étrangères et qu'effecti-

59

60

61

Michel Alliot, Les résistances traditionnelles, in R.J.P.I.C. 1967, p. 245.

M. Debene, La Justice sans juge, d'hier à demain, in La Justice en Afrique, op. cit., p. 90.

Keba M'Baye, Le droit en déroute, liberté et ordre social, Neuchâtel, Ed. de la banomière 1969, p. 38, rapporté par $I$. Abiabagna, op. cit., p. 156.

62 G. Pontie et M. Pilon, Un exemple de justice au quotidien: les conflits matrimoniaux au Nord-Togo, in La Justice en Afrique, op. cit., p. 104. 
vement elles ne correspondent pas à leur conception du droit et de la justice. Car, la justice africaine est fondamentalement différente de celle de l'Occident dont on a hérité. Le chef, contrairement au juge, ne cherche pas à trancher le noeud gordien du litige, en s'appuyant sur un texte figé mais plutôt à le dénouer en tentant de trouver un compromis acceptable pour les deux parties. "le rôle du juge ...", note Mgr De Clercq, "vise ... très souvent exclusivement à rétablir l'équilibre momentanément troublé ou rompu, par des solutions pratiques qui concilient les intérêts en cause, satisfassent si possible les deux parties, apaisent les adversaires; un bon juge s'évertue à convaincre de son tort la partie qui de fait a tort" ${ }^{63}$. L'on comprend dès lors que le rejet du juge moderne amène les justiciables africains à rechercher d'autres modes de solution de conflits qui, sans être strictement les mêmes que ceux de leurs ancêtres, s'en rapprochent, faisant appel à la médiation, à l'arbitrage ou à la conciliation. On aboutit à une justice négociée, à une "nouvelle justice sans juge" ${ }^{64}$ au sens Occidental du terme. Est-ce un retour aux sources?

En définitive l'obstacle majeur à l'accès à la justice réside dans la situation conflictuelle permanente des droits traditionnels et du droit moderne et dans le défaut d'effectivité de celui-ci autant d'ailleurs que dans la désagrégation progressive de ceux-là. Quelle solution proposée pour le surmonter? Il n'a a certes pas de recette miracle. Mais, pour franchir ce rubicon juridique l'uniformisation du droit allant dans le sens d'un nouveau droit, qui se veut synthétique, voire symbiotique, s'impose. Pour que ce nouvel ordre juridique africain s'applique effectivement il doit répondre à la fois aux exigences du développement économique et aux aspirations profondes des populations rurales, car les unes ne peuvent aller sans les autres étant indissociablement liées.

A. De Clercq, Le Ndumbulula, c'est à dire le juge en pays du Luba-Kasaï, Sankuru-Lomanu, Congo, tome 2, n⿳4 4, 1929, pp. 839, 844, rapporté par Mutoy Mubiala, L'individu devant la justice au Zaïre, 64 in La Justice, op. cit., pp. 185-186.

Voir dans ce sens M. Debene, in Justice, op. cit., p. 90. 


\section{ABSTRACTS}

\section{South Africa's New Constitution: the Challenges of Diversity and Identity}

\section{By Heinz Klug}

The state's imposition of racial classification under apartheid made racial identity the primary political identity for most South Africans. However, during the anti-apartheid struggle and to an even greater degree during the transition towards a post-apartheid era, South Af ricans discovered that political participation is built on a far more complex, fluid set of identities and interests.

\section{Access to Justice and Related Obstacles in the French-Speaking Sub-Saharan Africa}

\section{By René Degni-Segui}

Access to justice is perceived as a fundamental right, i.e. the right to resort to judiciary power. This right is all the more important as it constitutes the condition for other rights to justice. For without a trial an accused person can not def end himself and his rights.

The application in French-speaking sub-saharan Africa of the above-mentioned right, inherited from French law, is influenced by two parameters acting in opposite directions. One of them is favourable and linked to the guarantee that this right is exercised while the other is unfavourable and leads to an application which generates clashes with African rights. This conflicting situation creates obstacles to access to justice which are related to both law and context.

Obstacles related to laws are due to

a) the fact that many defendants find themselves far away from a court. Indeed, efforts have been made by African legislators to move the justice system closer to defendants through the creation of a number of jurisdictions. Not all states have agreed to such efforts, however, and the jurisdictions which have actually been set up have not been operational.

b) the slowness of the legal proceedings which, for political and cultural reasons, may extend over several years, sometimes up to 10 years.

c) the cost of lawsuit, considering that the costly legal proceedings are not compensated for by legal counseling which still remains very limited. 
Obstacles related to context are due to

a) the African political system which is characterized by the following factors:

- the authoritarianism of the African regimes prevents defendants from suing the administrative authorities for fear of retaliation so that they rather seek to settle conflicts through negociation.

- the justice system, subservient to the political power and the power of money, thus losing all credibility in the eyes of the African populations because of its partiality and vulnerability.

- the ineffectiveness of the institutions, as a result of the adoption by the African countries of western-style "modern" institutions, confining them to the museum of legal institutions.

b) Misappreciation of the foreign law transplanted to Africa takes on a double aspect:

- ignorance of the above-mentioned law by those Africans who are expected not to ignore it by virtue of the maxim "nemo legem ignorare censetur".

- refusal by the same persons to resort to state courts as the western justice concept is totally different from that of Africa based on the idea of conciliation.

Finally, and in order to cross this legal Rubicon, it seems essential to move towards a new symbiotic law capable of meeting both the requirements of economic development and the deep aspirations of the African populations.

\title{
The Transition to Multiparty Democracy in Tanzania: Some History and Missed Opportunities
}

\author{
By K. I. Tambila
}

After twenty-eight years of one-party rule, the constitution of Tanzania was amended in July 1992 to allow other parties to be formed. The amendment was made after a countrywide study of the mood and wishes of the Tanzanian people was undertaken by a Presidential Commission headed by the country's Chief Justice, Francis Nyalali.

Tanzania went through a short period of multi-party politics in the pre-independence period and an even shorter phase of two years after independence. Because of the colonial heritage which was undemocratic and also because of a widely accepted developmentalist ideology, among many other reasons, authoritarianism was easily resorted to by the nationalist leaders who inherited the colonial state and its apparatus. Developmentalism was also widely accepted by the Tanzanian populace who wanted quick and visible development denied it by the British and the Germans before them. In due course the ideology resulted in stifling the very development which was being pursued and created a 